\title{
NOTA SOBRE ENVIDIA, EQUILIBRIO Y OPTIMALIDAD *
}

En la presentación de Malinvaud de los Efectos Externos en el Consumo, (1), dado un modelo con dos individuos, dos bienes, un factor escaso y coeficientes de producción unitarios, se afirma que reducciones en el consumo del bien de lujo —envidiado- aumentan los niveles de utilidad de ambos individuos a partir del equilibrio considerado. Esto no parece obvio pues se desconoce la forma en que varían las utilidades y desutilidades marginales.

Demostramos aquí —aún para coeficientes de producción distintos de la unidad - la suficiencia de las características del equilibrio para determinar el efecto aludido sobre la situación de ambos agentes. Damos otra condición suficiente, que no supone necesariamente el equilibrio; generalizamos los resultados a un número mayor de consumidores y deducimos propiedades del óptimo de Pareto, definido como aquella situación en la que no es posible mejorar el nivel de utilidad de un agente sin reducir el de algún otro.

1. Consideremos una economía con dos consumidores $(\mathrm{j}=1,2)$ y dos bienes $(\mathrm{h}=1,2)$ tal que un estado posible dẹbe cumplir la condición:

$\mathrm{r}_{1} \mathrm{x}_{1}+\mathrm{r}_{2} \mathrm{x}_{2}=\mathrm{w}$, donde, $x_{h}=\sum_{j} x_{j h} ;$

$\mathrm{x}_{\mathrm{jh}}$, consumo del agente $\mathrm{j}$ del bien $\mathrm{h}$;

$\overline{\mathrm{w}}$, cantidad disponible $\overline{\text { del }}$ único $\overline{\text { factor escaso; }}$

$\overline{\mathrm{r}}_{\mathrm{h}}$, cantidad del bien escaso necesaria para producir una unidad de h.

* Deseamos agradecer el estímulo y los valiosos comentarios del Profesor Dr. Julio $\mathrm{H}$. G. Olivera, sin los cuales esta nota no se hubiera redactado. Los posibes errores subsistentes son de nuestra total responsabilidad.

(1) Malinvaud, E., Lecciones de teoría microeconómica, trad. A. Ortí Lahoz, Barcelona, Ed. Ariel, 1974, págs, 252 y 253. 
Por lo tanto, la tasa marginal de sustitución técnica resultará:

$$
\frac{-\mathrm{dx}_{2}}{\mathrm{dx}_{1}}=\frac{\mathrm{r}_{1}}{\mathrm{r}_{2}} \text {. }
$$

El súbíndice 2 identifica al bien de lujo. Su consumo genera efectos externos entre los consumidores; formalmente, si las funciones de utilidad de los individuos tienen las características de regularidad habituales, podemos expresarlas:

$$
\begin{aligned}
& \mathrm{S}_{1}=\mathrm{S}_{1}\left(\mathrm{x}_{11}, \mathrm{x}_{12}, \mathrm{x}_{22}\right), \\
& \mathrm{S}_{2}=\mathrm{S}_{2}\left(\mathrm{x}_{21}, \mathrm{x}_{22}, \mathrm{x}_{12}\right),
\end{aligned}
$$

ambas con derivadas negativas respecto del último argumento. Designando los opuestos de estas derivadas como $Q_{1}$ y $Q_{2}$ se verificará entonces:

$$
\text { (1) } Q_{1}>0, Q_{2}>0 \text {. }
$$

El óptimo de Pareto queda definido por:

$$
\frac{\mathrm{S}_{12}}{\mathrm{~S}_{11}}-\frac{\mathrm{Q}_{2}}{\mathrm{~S}_{21}}=\frac{\mathrm{S}_{22}}{\mathrm{~S}_{21}}-\frac{\mathrm{Q}_{1}}{\mathrm{~S}_{11}}=\frac{\mathrm{r}_{2}}{\mathrm{r}_{1}} ;
$$

mientras que el equilibrio, cuando cada consumidor toma como dado el consumo del bien de lujo del otro, es:

$$
\text { (2) } \frac{\mathrm{S}_{12}}{\mathrm{~S}_{11}}=\frac{\mathrm{S}_{22}}{\mathrm{~S}_{21}}=\frac{\mathrm{r}_{2}}{\mathrm{r}_{1}}=\frac{\mathrm{p}_{2}}{\mathrm{p}_{1}} \text {, }
$$

correspondiendo la última igualdad al equilibrio en la producción.

Se supone que: $\mathrm{x}_{12}>0, \mathrm{x}_{22}>0$, y que además, la variación en el consumo del bien de lujo es suficientemente pequeña.

Según Malinvaud, "en un equilibrio tal, el consumo del bien de lujo es demasiado elevado: se mejoraría el nivel de utilidad de los dos individuos si redujesen simultáneamente y de manera adecuada su consumo del bien 2 en beneficio del bien l". La primera parte de esta observación se comprueba notando que:

$$
\text { (3) } \frac{\mathrm{S}_{12}}{\mathrm{~S}_{11}}-\frac{\mathrm{Q}_{2}}{\mathrm{~S}_{21}}<\frac{\mathrm{r}_{2}}{\mathrm{r}_{1}} \text { y } \frac{\mathrm{S}_{22}}{\mathrm{~S}_{21}}-\frac{\mathrm{Q}_{1}}{\mathrm{~S}_{11}}<\frac{\mathrm{r}_{2}}{\mathrm{r}_{1}} \text {, }
$$

en virtud de (1) y (2). Además, las condiciones (1) son suficientes para el aumento simultáneo de las utilidades, como se expresa en la segunda parte del pasaje transcripto. Para verlo definamos:

$$
\begin{array}{cl}
\mathrm{dx}_{11}=\alpha \mathrm{dx}_{1}, & \mathrm{dx}_{21}=(1-\alpha) \mathrm{dx}_{1}, \\
\mathrm{dx}_{12}=\beta \mathrm{dx}_{2}, & \mathrm{dx}_{22}=(1-\beta) \mathrm{dx}_{2}, \\
\text { donde } 0 \leqslant \alpha, \beta \leqslant 1 \text { y además } \mathrm{dx}_{1}>0 .
\end{array}
$$


Diferenciando las funciones de utilidad en el equilibrio:

$$
\begin{aligned}
& \mathrm{dS}_{1}=\left\{\alpha \mathrm{S}_{11}+\frac{\mathrm{r}_{1}}{\mathrm{r}_{2}}\left((1-\beta) \mathrm{Q}_{1}-\beta \mathrm{S}_{12}\right)\right] \mathrm{dx}_{1}, \\
& \mathrm{dS}_{2}=\left[(1-\alpha) \mathrm{S}_{21}+\frac{\mathrm{r}_{1}}{\mathrm{r}_{2}}\left(\beta \mathrm{Q}_{2}-(1-\beta) \mathrm{S}_{22}\right)\right] \mathrm{dx}_{1},
\end{aligned}
$$

Haciendo $\alpha=\beta$ se cumplen por (2):

$$
\begin{gathered}
\mathrm{dS}_{1}=\frac{\mathrm{r}_{1}}{\mathrm{r}_{2}}(1-\beta) \mathrm{Q}_{1} \mathrm{dx}_{1}>0, \\
\mathrm{dS}_{2}=\frac{\mathrm{r}_{1}}{\mathrm{r}_{2}} \quad \beta \mathrm{Q}_{2} \mathrm{dx}_{1}>0 .
\end{gathered}
$$

Obsérvese que éstas son independientes de la forma en que estén variando las utilidades y desutilidades marginales (dado $-\mathrm{r}_{1} \mathrm{dx}_{1} / \mathrm{r}_{2}<0$ ).

2. Es interesante notar que, para que exista una distribución $\beta$ de la caída del consumo del bien de lujo, que mejore por lo menos el nivel de utilidad de un individuo (sin empeorar el del otro), basta que en el punto considerado:

$$
\mathrm{Q}_{1} \mathrm{Q}_{2} \geqslant \mathrm{~S}_{12} \mathrm{~S}_{22} \text {. }
$$

En efecto, si es así, puede tomarse un $\beta$ tal que:

$$
\frac{S_{12}}{Q_{1}} \leqslant \frac{1-\beta}{\beta} \leqslant \frac{Q_{2}}{S_{22}},
$$

de donde:

$$
0<\frac{S_{22}}{S_{22}+Q_{2}} \leqslant \beta \leqslant \frac{Q_{1}}{Q_{1}+S_{12}}<1,
$$

con lo que se cumplirán al mismo tiempo:

$$
\begin{gathered}
\frac{\mathrm{dS}_{1}}{\mathrm{dx}_{1}}=\alpha \mathrm{S}_{11}+\frac{\mathrm{r}_{1}}{\mathrm{r}_{2}}\left\{\mathrm{Q}_{1}-\beta\left(\mathrm{Q}_{1}+\mathrm{S}_{12}\right)\right\} \geqslant \alpha \mathrm{S}_{11} \geqslant 0, y \\
\left.\frac{\mathrm{dS}_{2}}{\mathrm{dx}_{1}}=(1-\alpha) \mathrm{S}_{21}+\frac{\mathrm{r}_{1}}{\mathrm{r}_{2}} \mid-\mathrm{S}_{22}+\beta\left(\mathrm{S}_{22}+\mathrm{Q}_{2}\right)\right\} \geqslant(1-\alpha) \mathrm{S}_{21} \geqslant 0 .
\end{gathered}
$$


Adviértase que una condición necesaria para la proposición es que ६в ] 0,1 [, es decir, ninguno de los individuos deja de participar en $\mathrm{dx}_{2}$.

Además, ambas desigualdades no pueden ser simultáneamente débiles, pues $\alpha \varepsilon[0,1]$.

Un coro'ario de la proposición que acabamos de verificar es que en el óptimo de Pareto se cumple:

$$
Q_{1} Q_{2}<S_{12} S_{22}
$$

Si no fuera así, existiría una distribución de la caída del consumo del bien 2 que permitiría aumentar la utilidad de por lo menos uno de los individuos sin a'terar la del otro.

3. Generalizamos ahora el modelo a! caso en el que la economía cuenta con $\mathrm{n}$ consumidores. Definimos:

$\mathrm{S}_{\mathrm{jh}}$, derivadla de la función de utilidad del individuo $\mathrm{j}$ con respecto a su consumo del bien h.

$\underline{Q}^{j}$ ih, derivada de la función de utilidad del agente $\mathrm{j}$ con respecto al consumo del bien $\mathrm{h}$ por el individuo $\mathrm{i}$.

$$
\begin{aligned}
& \alpha_{j}=\frac{\mathrm{dx}_{\mathrm{j}_{1}}}{\mathrm{dx}_{1}} . \\
& \beta_{\mathrm{j}}=\frac{\mathrm{dx}_{\mathrm{j} 2}}{\mathrm{dx}_{2}} . \\
& (\mathrm{h}=1,2) \\
& (\mathrm{i}, \mathrm{j}=1, \ldots, \mathrm{n})
\end{aligned}
$$

Obtenemos las siguientes proposiciones:

1) Condición suficiente para que las distribuciones $\left(\alpha_{1}, \ldots \alpha_{n}\right)$ y $\left(\beta_{1}, \ldots \beta_{n}\right)$ originen mejoras en los niveles de utilidad, a partir del equilibrio, es que para todo $\mathrm{j}$ se dé $\alpha_{\mathrm{j}}=\beta_{\mathrm{j}}$.

$$
\text { En efecto, si } \alpha_{\mathrm{j}}=\beta_{\mathrm{j}} \text { entonces }
$$

(1.1)

$$
\begin{aligned}
& \sum_{i \neq j} \frac{\beta_{i}}{S^{j}{ }_{j 2}^{j}} \geqslant \beta_{j}-\alpha_{j}, \\
& (\mathrm{j} \neq 1, \ldots, \mathrm{n})
\end{aligned}
$$


pues

$\mathrm{Q}^{\mathrm{j}}{ }_{\mathrm{i}} \geqslant 0$.

Como en el equilibrio:

$$
\mathrm{S}_{\mathrm{j} 2}=\frac{\mathrm{r}_{2}}{\mathrm{r}_{1}} \mathrm{~S}_{\mathrm{j} 1},
$$

y la variación de la utilidad es:

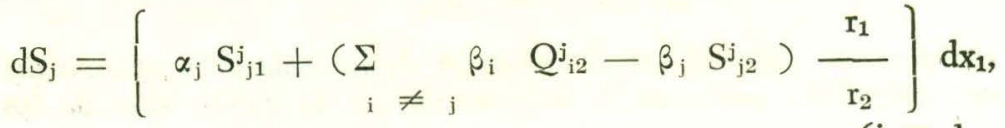

$$
\begin{aligned}
& (\mathrm{j}=1, \ldots, \mathrm{n})
\end{aligned}
$$

puede escribirse:

$$
\begin{aligned}
\frac{\mathrm{dS}_{\mathrm{j}}}{\mathrm{S}_{\mathrm{j} 2}^{\mathrm{j}}}=\left[\alpha_{\mathrm{j}}+{ }_{\mathrm{i}}^{\Sigma} \neq{ }_{\mathrm{j}} \quad \beta_{\mathrm{i}} \frac{\mathrm{Q}_{\mathrm{i} 2}^{\mathrm{j}_{2}}}{\mathrm{~S}_{\mathrm{j} 2}}-\beta_{\mathrm{j}}\right) & \frac{\mathrm{r}_{1}}{\mathrm{r}_{2}} \mathrm{dx}_{1}, \\
& (\mathrm{j}=1, \ldots, \mathrm{n})
\end{aligned}
$$

positiva o nula por (1.1) ya que $\mathrm{S}_{j 2}{ }_{j 2}>0$, y positiva para a gún $\underline{j}$ si

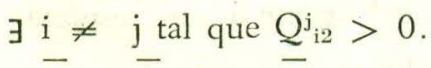

2) Si se cumple la condición:

$$
\Sigma \operatorname{máx}_{\mathrm{i}}\left\{\frac{\mathrm{S}_{\mathrm{j} 2}}{\mathrm{Q}_{\mathrm{i} 2}}\right\} \leqslant \mathrm{n}-1
$$

existe una distriłbución en la reducción del consumo del bien de lujo tal que aumentan simultáneamente los niveles de utilidad de todos los consumidores.

Efectivamente, en tal caso pueden tomarse para todo i:

$$
\beta_{i} \geqslant \frac{1}{n-1} \operatorname{máx}_{i}\left\{\frac{S_{j 2}^{j_{j}}}{Q^{j}{ }_{i 2}}\right\},
$$

tales que dichas $\beta_{i}$ sumen 1 . Se advierte enseguida que, entonces:

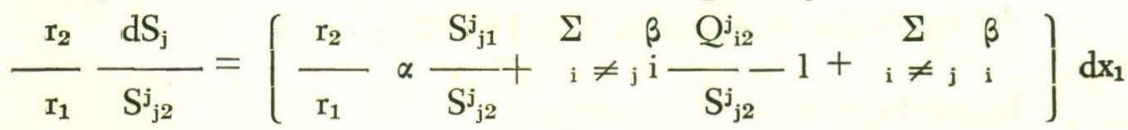
serán positivas pues:

$$
\frac{1}{\mathrm{n}-1} \operatorname{máx}_{\mathrm{i}}\left\{\frac{\mathrm{S}_{\mathrm{j} 2}}{\mathrm{Q}_{\mathrm{i} 2}}\right\} \frac{\mathrm{Q}_{\mathrm{i} 2}}{\mathrm{~S}_{\mathrm{j} 2}} \geqslant \frac{1}{\mathrm{n}-1}
$$


Un corolario es que en el óptimo de Pareto:

$$
\Sigma_{\mathrm{j}} \operatorname{máx}\left\{\frac{\mathrm{S}_{\mathrm{j} 2}}{\mathrm{Q}_{\mathrm{i} 2}^{\mathrm{j}}}\right\}>\mathrm{n}-1
$$

Si así no fuera se aplicaría la proposición precedente contradiciendo la definición del óptimo.

Adviértase que en $\mathrm{e}^{\mathrm{T}}$ caso de dos agentes:

$$
\frac{\mathrm{S}^{1}{ }_{12}}{\mathrm{Q}^{1}{ }_{22}}+\frac{\mathrm{S}^{2}{ }_{22}}{\mathrm{Q}^{2}{ }_{12}} \leqslant 1 .
$$

Como ambos términos son positivos y por lo tanto menores que uno,

$$
\mathrm{S}^{1}{ }_{12} \quad \mathrm{~S}_{22}^{2} \leqslant \mathrm{Q}^{1}{ }_{22} \quad \mathrm{Q}^{2}{ }_{12} \quad \mathrm{Q}^{2}{ }_{12},
$$

que es la condición enunciada en la Sección 2.

En resumen, en su presentación de los Efectos Externos en el Consumo, dado un modelo con dos individuos, dos bienes, un factor escaso y coeficientes de producción unitarios, Malinvaud afirma que reducciones en el consumo del bien de lujo - envidiado- aumentan los niveles de utilidad de ambos individuos a partir del equilibrio considerado. Esto no parece obvio pues se desconoce la forma en que varían las utilidades y desutilidades margina'es.

Se demuestra en esta nota —aún para coeficientes de producción distintos de la unidad - la suficiencia de las características del equilibrio para determinar el efecto aludido sobre la situación de ambos agentes. Se enuncia otra condición suficiente, que no supone necesariamente el equilibrio, pero cuyo cumplimiento depende del número de agentes de la economía; los resultados se generalizan a un número mayor de consumidores y se deducen propiedades del óptimo de Pareto.

\section{Cristina, Marcela y Chisari, Omar Universidad de Buenos Aires}

